\title{
CARDIAC SAFETY
}

\section{Lomitapide at Supratherapeutic Plasma Levels Does Not Prolong the QTc Interval-Results from a TQT Study with Moxifloxacin and Ketoconazole}

\author{
Borje Darpo, M.D. Ph.D., ${ }^{*} \dagger$ Georg Ferber, Ph.D. $\neq$ Meijian Zhou, Ph.D., ${ }^{*}$ \\ Mark Sumeray, M.D., $\S$ and Philip Sager, M.D. $\uparrow$
}

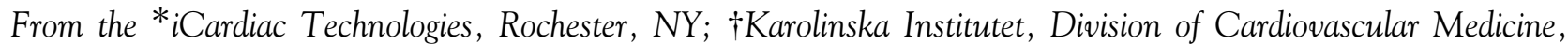
Department of Clinical Sciences, Danderyd's Hospital, Stockholm, Sweden; $\$$ Statistik Georg Ferber GmbH, Riehen, Switzerland; §Aegerion Pharmaceuticals, Cambridge, MA; and \$Sager Consulting Experts, Inc, San Francisco, CA

Background: The aim of this study was to assess the effect of high plasma levels of lomitapide and its main metabolite on ECG parameters.

Methods: In this randomized five-way cross-over thorough QT study, 56 healthy subjects were enrolled. Study treatments were administered orally for 3 days in five separate periods in which subjects were dosed with (1) a single dose of $75 \mathrm{mg}$ lomitapide on Day 1 followed by a single dose of $200 \mathrm{mg}$ on Day 3; (2) ketoconazole $200 \mathrm{mg}$ BID; (3) ketoconazole with a single dose of $75 \mathrm{mg}$ lomitapide on Day 3; (4) a single dose of 400 mg moxifloxacin on Day 3 and (5) placebo.

Results: Single doses of 75 and $200 \mathrm{mg}$ lomitapide alone or in combination with ketoconazole caused minor changes in the change-from-baseline QTCl $(\Delta \mathrm{QTCl})$, whereas moxifloxacin and ketoconazole caused an increase of $\Delta$ QTCl with a peak effect at 1 and 3 hours postdosing, respectively. The largest mean placebo-corrected $\Delta \mathrm{QTCl}(\Delta \Delta \mathrm{QTCl})$ for lomitapide did not exceed $3 \mathrm{~ms}$ (upper bound of $90 \% \mathrm{Cl}: 4.7 \mathrm{~ms}$ ) at any time points postdosing. Ketoconazole caused mild QT prolongation with mean $\Delta \Delta \mathrm{QTCl}$ of 5.9 and $6.5 \mathrm{~ms}$ at 2 and 3 hours postdosing, and exposureresponse analysis demonstrated a significantly positive slope of $1.3 \mathrm{~ms}$ per $\mu \mathrm{g} / \mathrm{mL}(90 \% \mathrm{Cl}: 1.0-1.7)$. Moxifloxacin met the criteria for assay sensitivity.

Conclusions: Lomitapide does not have an effect on cardiac repolarization. The study's ability to detect small QTc changes was demonstrated with both moxifloxacin and ketoconazole.

Ann Noninvasive Electrocardiol 2013;18(6):577-589

$\underline{\text { lomitapide; QT/QTc; thorough QT study; ketoconazole; healthy volunteers }}$

Lomitapide is an orally effective, selective inhibitor of microsomal triglyceride transfer protein (MTP), which has recently been approved in the United States for the treatment of patients with homozygous familial hypercholesterolemia (HoFH). Inhibition of MTP prevents the assembly of apoBcontaining lipoproteins in hepatocytes and enterocytes and limits the release of these lipoproteins into the systemic circulation. These characteristics make lomitapide a promising agent for patients with specific genetic disorders, which result in hypercholesterolemia, hypertriglyceridemia, and/or mixed lipid disorders involving both cholesterol and triglycerides.

Since the adoption of the International Conference of Harmonisation (ICH) efficacy guidance E14 in $2005{ }_{1}{ }^{1}$ there is a regulatory recommendation to study the effect of all new chemical entities on the surface ECG QT interval in a specifically designated study, the thorough QT/QTc (TQT)

Address for correspondence: Borje Darpo, M.D., Ph.D., Karolinska Institutet, Division of Cardiovascular Medicine, Department of Clinical Sciences, Danderyd's Hospital, Stockholm, Sweden. Fax: +46 876616 32; E-mail: borje.darpo@telia.com

Financial support: This conduct and analysis of this study was funded by Aegerion Pharmaceuticals, Cambridge, MA 02142, USA. 
study. This study is normally performed in healthy volunteers, who are exposed to high plasma levels of the drug and a negative (placebo) and a positive control, in most cases moxifloxacin. ${ }^{2}$ Initially perceived as a great challenge, ${ }^{3}$ the TQT study is today performed routinely as part of clinical development and almost 300 studies have been reviewed by the FDA by the end of 2012. Because the results of the TQT study impact the level of ECG monitoring in subsequent trials, ${ }^{1,4}$ it is often performed before the initiation of Phase 3 studies.

An important feature that needs to be addressed when designing a TQT study is the selection of the supratherapeutic dose. This dose should generate plasma levels of the parent drug and abundant metabolites, which clearly exceed those that can be observed in patients with impaired clearance of the drug, based on intrinsic (e.g., age and hepatic impairment) or extrinsic factors, specifically food and drug interactions. Lomitapide is a CYP $3 \mathrm{~A} 4$ substrate and patients concomitantly administered a strong 3A4 inhibitor, such as ketoconazole, will have several-fold higher plasma levels of the parent compound, whereas metabolites will appear in substantially lower than normal concentrations (data on file, Aegerion Pharmaceuticals, Cambridge, MA, USA). It was therefore considered appropriate to perform the TQT study with lomitapide with one treatment period in which lomitapide was given together with ketoconazole, in addition to a therapeutic and a supratherapeutic dose. The supratherapeutic dose permitted the evaluation of high levels of lomitapide metabolites in addition to the parent compound, whereas ketoconazole coadministered with lomitapide resulted in high exposures of the parent compound.

It has been demonstrated that 24 hours of preexposure to ketoconazole (200 mg every 12 hours before administration of the substrate) is sufficient to attain maximum CYP3A inhibition with no further effect with greater duration. ${ }^{5}$ Accordingly, ketoconazole was given for 2 days, including a 1-day run-in treatment with BID administration every 12 hours, followed by coadministration of lomitapide and ketoconazole.

Because ketoconazole itself also causes mild QT prolongation, ${ }^{6-9}$ a separate period with only ketoconazole was also included to allow for adjustment of the combined lomitapide-ketoconazole effect.

\section{METHODS}

\section{Study Design}

This was a single-center, randomized, fiveperiod, cross-over study. In separate 3-day treatment periods, subjects received single doses of lomitapide 75 and $200 \mathrm{mg}, 75 \mathrm{mg}$ lomitapide concomitantly with $200 \mathrm{mg}$ ketoconazole (Nizoral ${ }^{\circledR}$ ), (single dose) or placebo (Table 1). Subjects resided at the clinical site from the afternoon of the day before dosing (Day -1) until Day 5, 48 hours after the last dose administration on Day 3. Each period was separated by a 10 days washout period. All treatments were given as oral formulations; lomitapide and placebo as solutions and moxifloxacin and ketoconazole as tablets. Doses on Days 1 and 3 were administered following a light breakfast snack served 1 hour 15 minutes before dosing, followed by a fast from food for at least 4 hours postdose. The study was double-blinded with regard to the lomitapide and placebo treatments, and open label for the ketoconazole and moxifloxacin treatments. The ECG laboratory was blinded to all study treatments.

Fifty-six, nonsmoking healthy male and female subjects between 18 and 55 years of age (inclusive) were to be included with a target proportion of at least $33 \%$ of each gender. Exclusion criteria included baseline heart rate $<45 \mathrm{bpm}$ or $>100 \mathrm{bpm}$ and ECG abnormalities, e.g., QTc $>450 \mathrm{~ms}$ for males and $>470 \mathrm{~ms}$ for females.

\section{2-Lead Electrocardiogram Acquisition and Measurements}

Continuous 12-lead ECG recordings were performed on Day 1 and 3 of each treatment period using the Global Instrumentation M12R Holter device (Global Instrumentation, Buffalo, NY, USA) The ECGs were stored on a flash card and were not available for review until the card was received by the ECG laboratory. ECGs were extracted from the continuous recording on Days 1 and 3 of each treatment period with the use of proprietary software (TQTPlu ${ }^{\circledR}$ ) to optimize the quality of extracted waveforms, based on stable heart rate, a high signal-to-noise ratio and other prespecified quality metrics. ${ }^{10}$ Up to 10 ECGs were extracted from the last 5 minutes of the 15minute period of supine resting at the following 
A.N.E. • November 2013 • Vol. 18, No. 6 • Darpo, et al., • Lomitapide TQT Study • 579

Table 1. Treatments

\begin{tabular}{|c|c|c|c|}
\hline Treatment & Day 1 & Day 2 & Day 3 \\
\hline Ketoconazole & $\begin{array}{l}\text { Single dose of placebo } \\
\text { for lomitapide }\end{array}$ & $\begin{array}{l}\text { Ketoconazole } 200 \mathrm{mg} \\
\text { BID + single dose of } \\
\text { placebo for lomitapide }\end{array}$ & $\begin{array}{l}\text { Ketoconazole } 200 \mathrm{mg} \\
\text { BID + single dose of } \\
\text { placebo for lomitapide }\end{array}$ \\
\hline $\begin{array}{l}\text { Ketoconazole with } \\
\text { lomitapide } 75 \mathrm{mg}\end{array}$ & $\begin{array}{l}\text { Single dose of placebo } \\
\text { for lomitapide }\end{array}$ & $\begin{array}{l}\text { Ketoconazole } 200 \mathrm{mg} \\
\text { BID + single dose of } \\
\text { placebo for lomitapide }\end{array}$ & $\begin{array}{l}\text { Ketoconazole } 200 \mathrm{mg} \\
\text { BID + single dose of } \\
75 \mathrm{mg} \text { lomitapide }\end{array}$ \\
\hline Moxifloxacin & $\begin{array}{l}\text { Single dose of placebo } \\
\text { for lomitapide }\end{array}$ & $\begin{array}{l}\text { Single dose of placebo } \\
\text { for lomitapide }\end{array}$ & $\begin{array}{l}\text { Single dose of } 400 \mathrm{mg} \\
\text { moxifloxacin }+ \text { single } \\
\text { dose of placebo for } \\
\text { lomitapide }\end{array}$ \\
\hline Lomitapide & $\begin{array}{l}\text { Single dose of } 75 \mathrm{mg} \\
\text { lomitapide }\end{array}$ & $\begin{array}{l}\text { Single dose of placebo } \\
\text { for lomitapide }\end{array}$ & $\begin{array}{l}\text { Single dose of } 200 \mathrm{mg} \\
\text { lomitapide }\end{array}$ \\
\hline Placebo & $\begin{array}{l}\text { Single dose of placebo } \\
\text { for lomitapide }\end{array}$ & $\begin{array}{l}\text { Single dose of placebo } \\
\text { for lomitapide }\end{array}$ & $\begin{array}{l}\text { Single dose of placebo } \\
\text { for lomitapide }\end{array}$ \\
\hline
\end{tabular}

prespecified time points: Predose at -45 minutes, -30 minutes, and -15 minutes before to the first dose on Day 1 and $1,2,3,4,5,7,12$, and 24 hours postdose on Day1 and Day 3 .

The High Precision QT measurement technique (HPQT) was used to measure the QT and RR intervals on all beats classified as high-confidence in the 10 ECG replicates. ${ }^{10,11}$ The primary analysis lead was Lead II. All low confidence beats were reviewed manually and adjudicated using passfail criteria and the final QC was performed by a cardiologist and beats found acceptable were included in the analysis. Review of all ECGs for a particular subject was performed by the same reader at the ECG laboratory. The median QT and $\mathrm{RR}$ values from all measured beats within each extracted replicate was calculated, and then the mean of the medians from all available replicates at the nominal time point was used as the subject's reportable value at that time point. Measurements of PR and QRS intervals as well as categorical Twave morphology analysis were performed fully manually in three of the 10 ECG replicates with the highest signal-to-noise ratio at each time point.

\section{Statistical Analysis}

The population for the ECG analysis consisted of all subjects who received at least one dose of study medication, and had at least one pretreatment baseline ECG and one postdose ECG within the same treatment period. For the exposureresponse (ER) analysis, a time-matched plasma concentration was also necessary.
Individualized QTc (QTcI) was derived as follows: (1) QT/RR pairs from all nominal time points on Day 1 from all treatment periods in which placebo was administered on this day (i.e., all except the lomitapide period), were used to derive subjects' individual correction formula; (2) Based on QT/RR pairs from all subjects, QTcI was derived from a linear mixed effects model: for $\log (\mathrm{QT})$ with $\log (\mathrm{RR})$ as covariate with gender included as a fixed effect and subject included as a random effect for both intercept and slope. The $\log (R R)$ coefficient for each subject, $b_{i}$, was then used to calculate the individually corrected QT for each subject as follows: QTcI $=\mathrm{QT} / \mathrm{RR}^{\mathrm{bi}}$ - QT. Correction according to Fridericia's formula was defined as $\mathrm{QTcF}=\mathrm{QT} / \mathrm{RR} \cdot{ }^{1,3}$

For selection of the primary end point $(\mathrm{QTcF}$ or QTcI), the relationship between QTc and $\mathrm{RR}$ interval was investigated using on-treatment data by period using a linear regression model: QTc $=a+b_{l i} \times$ RR. Mean QTc and RR values from all nominal time points (including predose) were used. The RR coefficient for each subject, $b_{i}$, was used to calculate the sum of squared slopes (SSS) for each of the different QTRR correction methods as proposed by FDA's Interdisciplinary Review Team ${ }^{12}$. The correction method that resulted in the mean on-treatment slope closest to zero (the smallest mean SSS) was deemed the most appropriate heart rate correction method and was therefore used for the primary end point.

The primary end point was the change from baseline QTc corrected for the respective placebo or ketoconazole alone, respectively ( $\Delta \Delta \mathrm{QTC}$ ). 
Baseline was the mean of the 3 predose time points from Day 1 in each treatment period. $\Delta$ QTc was analyzed using a mixed effects model with the following covariates: time (categorical), treatment, time by treatment interaction, and the baseline value of the parameter. Because this was a crossover design, period and sequence terms were also included in the model. Subject was included as a random effect. A two-sided $90 \%$ confidence interval (CI) was calculated. The analysis was based on the Intersection Union Test. ${ }^{13,14}$ with the null hypothesis that the upper bound (UB) of the $90 \%$ $\mathrm{CI}$ for $\Delta \Delta \mathrm{QTC}$ exceeded $10 \mathrm{~ms}$ at at least one of the postdosing time points. Since the Intersection Union Test was applied, no adjustment for testing at multiple end points was needed. The UB of the 2 -sided $90 \%$ CI on treatment was compared to the $10 \mathrm{~ms}$ bound for lomitapide $75 \mathrm{mg}$ versus placebo on Day 1, for lomitapide $200 \mathrm{mg}$ versus placebo on Day 3 and for lomitapide + ketoconazole versus ketoconazole alone. To establish assay sensitivity, the lower confidence bound (adjusted for multiplicity using the Hochberg approach ${ }^{15}$ ) of the mean difference of moxifloxacin and placebo had to exceed $5 \mathrm{~ms}$ at least one of the prespecified time points: 1, 2, 3, and 4 hours. Because the largest mean $\Delta \Delta \mathrm{QTCI}$ for moxifloxacin was observed at the earliest prespecified time point after dosing (1 hour), three additional time points $(0.25,0.5$, and 0.75 hours postdosing) were analyzed post hoc descriptively for moxifloxacin and placebo to assess whether there was a rising phase in the moxifloxacin $\Delta \Delta \mathrm{QTC}$ response.

For categorical outliers, the number (percentage) of subjects and time points with increases in QTc (QTcF and QTcI) from baseline of $>30 \mathrm{~ms}$ and $>60$ $\mathrm{ms}$, and absolute QTc values $>450 \mathrm{~ms},>480 \mathrm{~ms}$, and $>500 \mathrm{~ms}$ was determined by treatment, respectively.

The relationship between $\Delta \Delta \mathrm{QTCI}$ and plasma concentrations of lomitapide and ketoconazole was investigated using a multivariate linear model as proposed by $\mathrm{Zhu}$ et al. ${ }^{9}$ Data were pooled from lomitapide-alone, ketoconazole-alone, and lomitapide plus ketoconazole treatment periods for analysis. The full model included separate slope parameters for lomitapide and ketoconazole effects. A parameter for the interaction of the two concentrations was also included..$^{9}$ Model details were as follows:

$$
\begin{aligned}
& \Delta \Delta \mathrm{QTCI}_{\mathrm{ij}}= \alpha_{\mathrm{i}}+\beta_{1 \mathrm{i}} \times \mathrm{C}_{\text {Lomiij }}+\beta_{2 \mathrm{i}} \times \mathrm{C}_{\text {Ketoij }} \\
&+\beta_{3 \mathrm{i}} \times \mathrm{C}_{\text {Lomiij }} \times \mathrm{C}_{\text {Ketoij }}+\varepsilon_{\mathrm{ij}} \\
&\left(\begin{array}{c}
\alpha_{\mathrm{i}} \\
\beta_{1 \mathrm{i}} \\
\beta_{2 \mathrm{i}} \\
\beta_{3 \mathrm{i}}
\end{array}\right) \sim \mathrm{MVN}\left(\left(\begin{array}{c}
\alpha \\
\beta_{1} \\
\beta_{2} \\
\beta_{3}
\end{array}\right),(\Omega)\right) \\
& \varepsilon_{\mathrm{ij}} \sim \mathrm{N}\left(0, \sigma^{2}\right),
\end{aligned}
$$

where $\mathrm{C}_{\text {Lomiij }}$ was the lomitapide concentration for $\mathrm{i}^{\text {th }}$ subject at the $\mathrm{j}^{\text {th }}$ time point, and $\mathrm{C}_{\text {Ketoij }}$ represented the ketoconazole concentration for the $\mathrm{i}^{\text {th }}$ subject and $\mathrm{j}^{\text {th }}$ time point. $\alpha_{\mathrm{i}}$ was the intercept for the $\mathrm{i}^{\text {th }}$ subject, and $\beta_{1 \mathrm{i}}, \beta_{2 \mathrm{i}}$, and $\beta_{3 \mathrm{i}}$ were three slopes representing the QT interval prolongation effect from lomitapide concentration, ketoconazole concentration, and the interaction from both lomitapide and ketoconazole concentrations for the $\mathrm{i}^{\text {th }}$ subject. The relationship between the population and individual parameters (i.e., slopes and intercept) was assumed to follow a multivariate normal distribution. Alfa $(\alpha)$ was the population mean intercept, and $\beta_{1}, \beta_{2}$, and $\beta_{3}$ were the population mean slopes for the QT effect from lomitapide concentration, ketoconazole concentration, and the interaction from both lomitapide and ketoconazole concentrations; $\Omega$ was the variance-covariance matrix for the population intercept and slopes assuming unstructured. $\varepsilon_{\mathrm{ij}}$ was the residual error for the $\mathrm{i}^{\text {th }}$ subject at the $\mathrm{j}^{\text {th }}$ observation, and it followed a normal distribution with a mean of 0 and a variance of $\sigma .^{2}$ A plot of standardized residuals versus fitted values was used to examine departure from model assumptions. The normal $\mathrm{Q}-\mathrm{Q}$ plots of the random effects and the within-subject errors were used to investigate the normality of the random effects and the within-subject errors, respectively. A final assessment of the adequacy of the linear mixed effects model was provided by a goodness-of-fit plot, proposed by the FDA's Interdisciplinary Review Team. ${ }^{12,16,17}$ The individual $\Delta \Delta$ QTCI values in 75 $\mathrm{mg}$ lomitapide coadministered with ketoconazole were adjusted for ketoconazole and lomitapideketoconazole interaction effects for plot over lomitapide concentrations, and adjusted for lomitapide and lomitapide-ketoconazole interaction 
effects for plot over ketoconazole concentrations. Such a plot was used to check the assumption of linearity between plasma concentrations of lomitapide and ketoconazole and $\Delta \Delta \mathrm{QTCI}$ and how well the predicted $\Delta \Delta$ QTcI matched the observed data in the regions of interest. The goodness-offit plot was generated by binning the independent variable (i.e., concentrations) into deciles. The mean $\Delta \Delta$ QTcI with $90 \%$ CI within each decile was computed and plotted at the corresponding median concentration within the decile.

\section{RESULTS}

Fifty-six (56) subjects were enrolled into the study; 52 completed the ketoconazole and placebo periods, 53 the moxifloxacin and lomitapide periods and 54 the lomitapide + ketoconazole period. Thirty-seven subjects $(66 \%)$ were male and $35(63 \%)$ were white and $32 \%$ were black or African American. The mean age of enrolled subjects was 38 years (standard deviation [SD] 9.4 years) and their mean body mass index was $26.3 \mathrm{~kg} / \mathrm{m}^{2}$ (SD: $2.20 \mathrm{~kg} / \mathrm{m}^{2}$ ).

\section{Plasma Levels}

Concentration-time profiles for lomitapide and ketoconazole are shown in Figure 1. The lomitapide geometric mean peak plasma concentration $\left(\mathrm{C}_{\max }\right)$ after a single oral solution dose of $75 \mathrm{mg}$ and $200 \mathrm{mg}$ and $75 \mathrm{mg}$ combined with ketoconazole reached $18 \mathrm{ng} / \mathrm{mL}(90 \%$ CI: $16.2-19.9)$ at a median of 3 hours, $66 \mathrm{ng} / \mathrm{mL}$ (90\% CI: 58.2-73.8) at 3 hours and $92 \mathrm{ng} / \mathrm{mL}(82.5-103.3)$ at 4 hours, respectively, i.e., metabolic inhibition with a potent CYP P450 3A4 inhibitor caused a fivefold increase in lomitapide $\mathrm{C}_{\max }$. Mean peak plasma levels of ketoconazole reached 5493 and $4998 \mathrm{ng} / \mathrm{mL}$ at a median of 2 and 2 hours in the ketoconazole alone and lomitapide + ketoconazole periods, respectively.

\section{Effect on Heart Rate}

The effect on heart rate after dosing across treatments is shown in Figure 2. The mean changefrom-baseline heart rate $(\Delta \mathrm{HR})$ followed the same diurnal pattern in all treatment periods with a small reduction from 1 hour to 4 hours postdosing and a peak effect of approximately 7-9 bpm at 5-7 hours postdosing. Mean $\triangle \mathrm{HR}$ across treatments was generally overlapping and the resulting placebocorrected $\Delta \mathrm{HR}(\Delta \Delta \mathrm{HR})$ was therefore small and did not exceed -2.6-1.5 bpm in any of the lomitapide treatment arms (data not shown).

\section{Effects on Cardiac Repolarization-the QTc Interval}

Individual QTcF/RR and QTcI/RR slopes were evaluated using on-treatment data from Day 3. Both correction methods resulted in relatively flat QTc/RR slopes, with a somewhat higher mean sum of squared individual slopes (SSS) observed on $200 \mathrm{mg}$ lomitapide as compared to other treatment periods and consistently somewhat smaller mean SSS with QTcI, which therefore was selected as the primary end point (Table 2).

The diurnal pattern of $\Delta$ QTc was similar across all treatment periods (Fig. 3). A single dose of 75or $200 \mathrm{mg}$ lomitapide caused minor changes of $\Delta$ QTcI across all time points. Moxifloxacin and ketoconazole (alone or in combination with lomitapide) caused an increase of $\Delta$ QTcI with a peak effect at 3 hours postdosing. After administration of moxifloxacin, $\Delta$ QTcI ranged between $7.7 \mathrm{~ms}$ and $12.7 \mathrm{~ms}$ from 1 to 5 hours postdosing and declined from 7 hours and onwards. Ketoconazole alone caused an increase of $\Delta$ QTcI of $5.6 \mathrm{~ms}$ to $7.5 \mathrm{~ms}$ at 2-5 hours postdosing. When ketoconazole was combined with lomitapide, $\Delta$ QTcI changes were of the same magnitude (4.8-6.3 ms), indicating the absence of a meaningful effect of lomitapide on the QTcI. The largest mean placebo-corrected $\Delta$ QTcI $(\Delta \Delta$ QTCI $)$ after administration of 75 or 200 mg lomitapide did not exceed $3 \mathrm{~ms}$ at any time points postdosing (Table 3) and the highest UBs of the $90 \% \mathrm{CI}$ were $3.0 \mathrm{~ms}$ and $4.7 \mathrm{~ms}$, respectively. Lomitapide + ketoconazole caused a largest mean ketoconazole-corrected $\Delta \mathrm{QTcI}$ ( $\Delta \Delta \mathrm{QTCI}$ ) effect of $2.3 \mathrm{~ms}$ (UB of CI: 4.4) 24 hours after dosing. The $\Delta \Delta$ QTcI after dosing of moxifloxacin confirmed the study's ability to detect a small QTc effect; the largest mean $\Delta \Delta$ QTCI of $12.4 \mathrm{~ms}$ was observed 1 hour after administration and the lower bound of the $90 \% \mathrm{CI}$ exceeded $5 \mathrm{~ms}$ at all prespecified time points $(1,2,3$, and 4 hours).Time points earlier than 1 hour confirmed that there was a rising phase of the moxifloxacin $\Delta \Delta \mathrm{QTCI}$ response. $\Delta \Delta \mathrm{QTcI}$ at time points after the peak effect was somewhat lower but remained significantly elevated during the full observation period of 24 hours. In the 

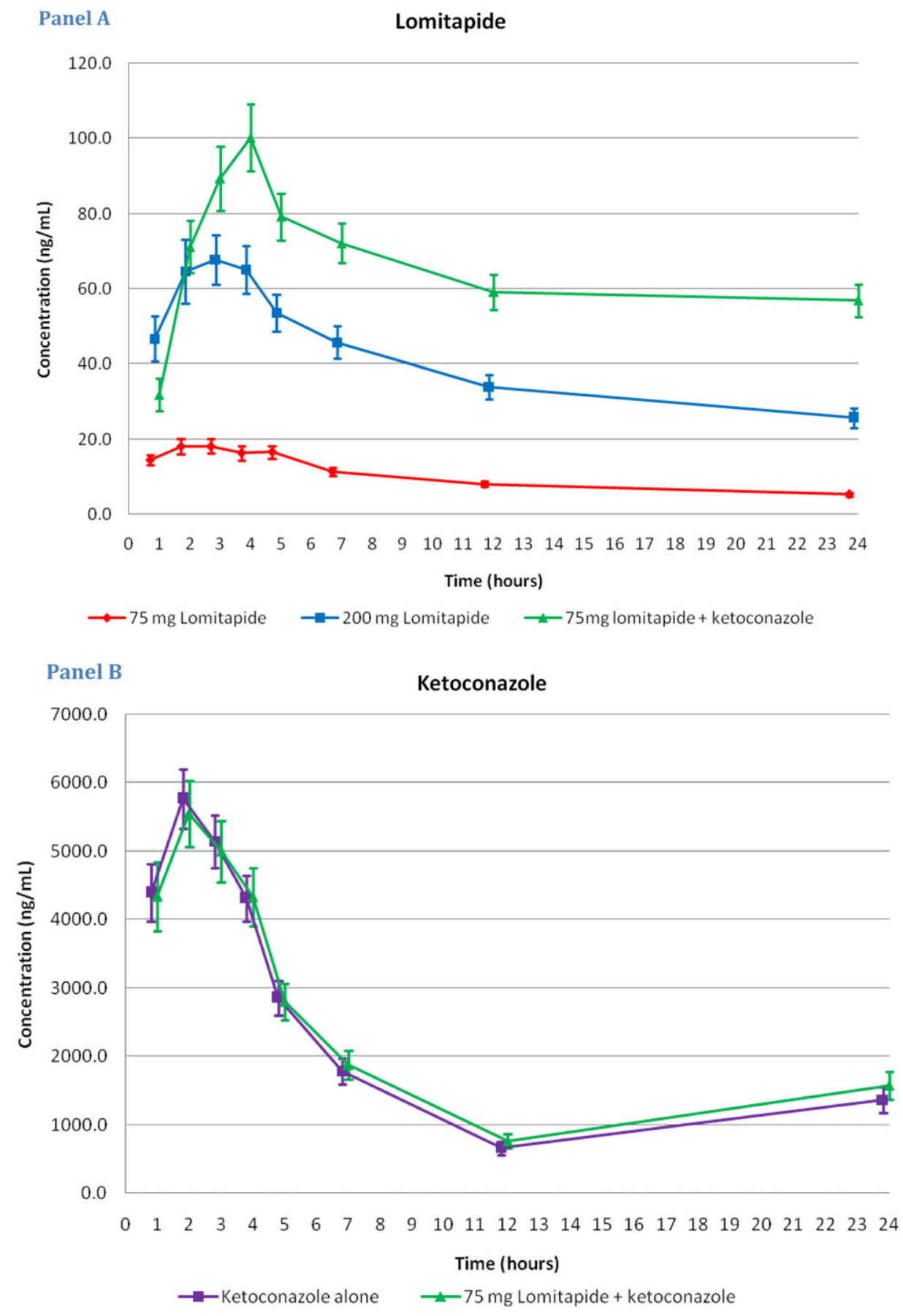

Figure 1. Concentration-time profiles for lomitapide (panel $A$; mean $\pm 90 \% \mathrm{Cl}$; $\mathrm{ng} / \mathrm{mL}$ ) and ketoconazole (panel $B$ ). Plasma levels of lomitapide increased fivefold with concomitant administration of a potent 3A4 inhibitor, ketoconazole. 


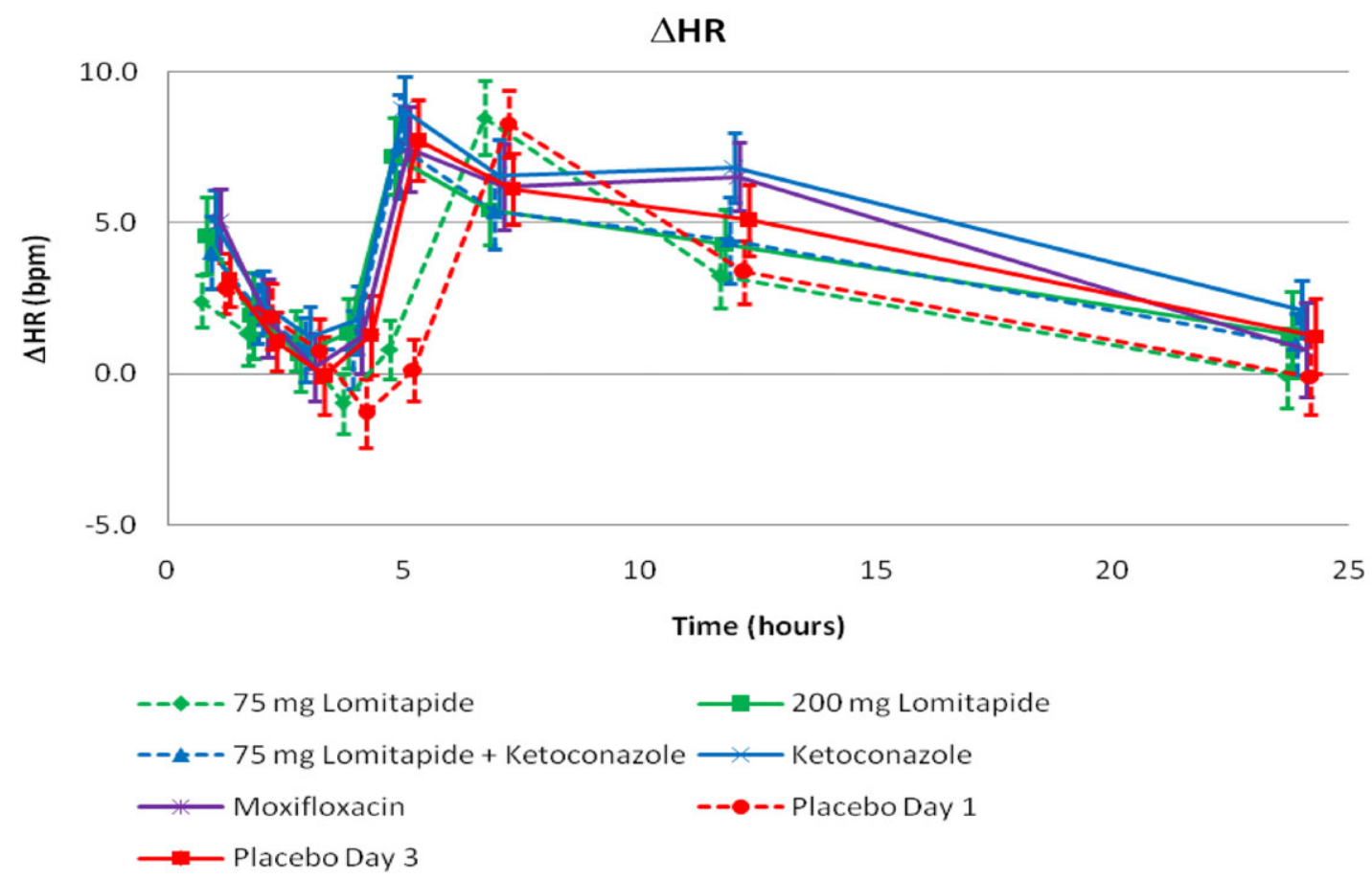

Figure 2. HR: Change from baseline $(\Delta \mathrm{HR})$ across treatment and time points based on summary statistics. A similar diurnal pattern was observed in all treatment periods. Lomitapide did not have an effect on heart rate.

Table 2. Evaluation of the Heart Rate Correction Method

\begin{tabular}{|c|c|c|}
\hline \multirow[b]{2}{*}{ Treatment } & \multicolumn{2}{|c|}{$\begin{array}{l}\text { Slope Estimates } \\
\text { Mean of Squared Individual Slopes }\end{array}$} \\
\hline & QTcF & QTcI \\
\hline $\begin{array}{l}200 \mathrm{mg} \text { lomitapide } \\
75 \text { mg lomitapide }+ \text { ketoconazole } \\
\text { Ketoconazole } \\
\text { Moxifloxacin } \\
\text { Placebo }\end{array}$ & $\begin{array}{l}0.0122 \\
0.0049 \\
0.0047 \\
0.0060 \\
0.0026\end{array}$ & $\begin{array}{l}0.0119 \\
0.0041 \\
0.0037 \\
0.0054 \\
0.0021\end{array}$ \\
\hline
\end{tabular}

ketoconazole alone treatment period, the largest $\Delta \Delta$ QTcI of $6.5 \mathrm{~ms}$ was observed at 3 hours and the lower bounds of the $90 \%$ CI exceeded 0 ms between 1 and 12 hours (Table 3). None of the subjects had a QTcI value exceeding $480 \mathrm{~ms}$ or a $\Delta$ QTcI exceeding $60 \mathrm{~ms}$ at any time postdosing in the 75 and $200 \mathrm{mg}$ lomitapide treatment period. Results from QTcF were entirely consistent with those obtained using QTcI (data not shown).

The precision of the QTc measurements measured as the SD of $\Delta$ QTCI was on average 6.6 ms across treatments and study days (Day 1 and 3).

\section{Effects on Cardiac Conduction-the PR and QRS Intervals}

The mean $\triangle \mathrm{PR}$ changes were very small without notable differences across treatments. The placebocorrected effect $(\Delta \Delta \mathrm{PR})$ was slightly prolonged in the $200 \mathrm{mg}$ lomitapide arm at 7 hours with a mean effect of $4.5 \mathrm{~ms}$ (90\% CI: 0.9-8.1 ms), whereas no such effect was observed when lomitapide was given with ketoconazole. Lomitapide did not affect the QRS interval; the largest mean $\Delta \Delta$ QRS after 75 or $200 \mathrm{mg}$ lomitapide or lomitapide with ketoconazole was $1.4 \mathrm{~ms}$ and the UB of the $90 \% \mathrm{CI}$ did not exceed $1.5 \mathrm{~ms}$ at any time point. 
Panel A

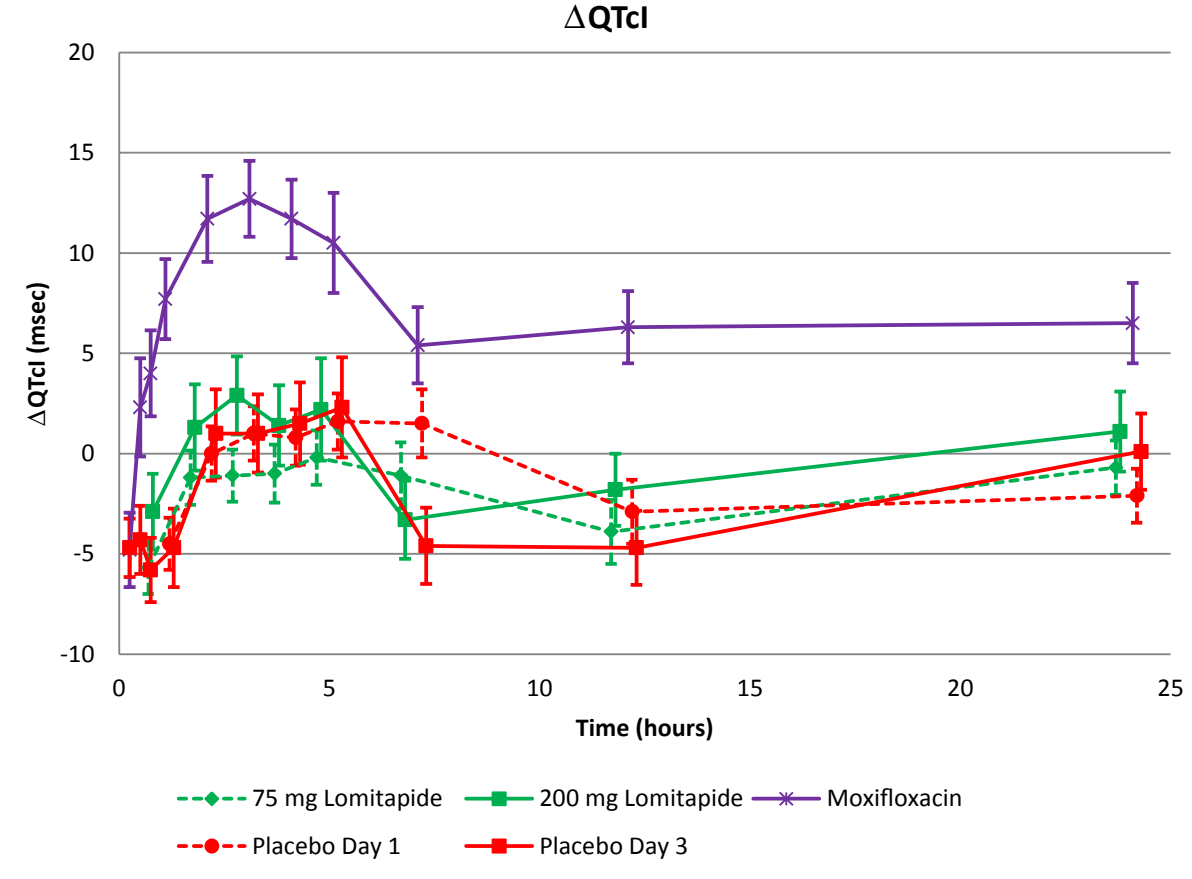

Panel B

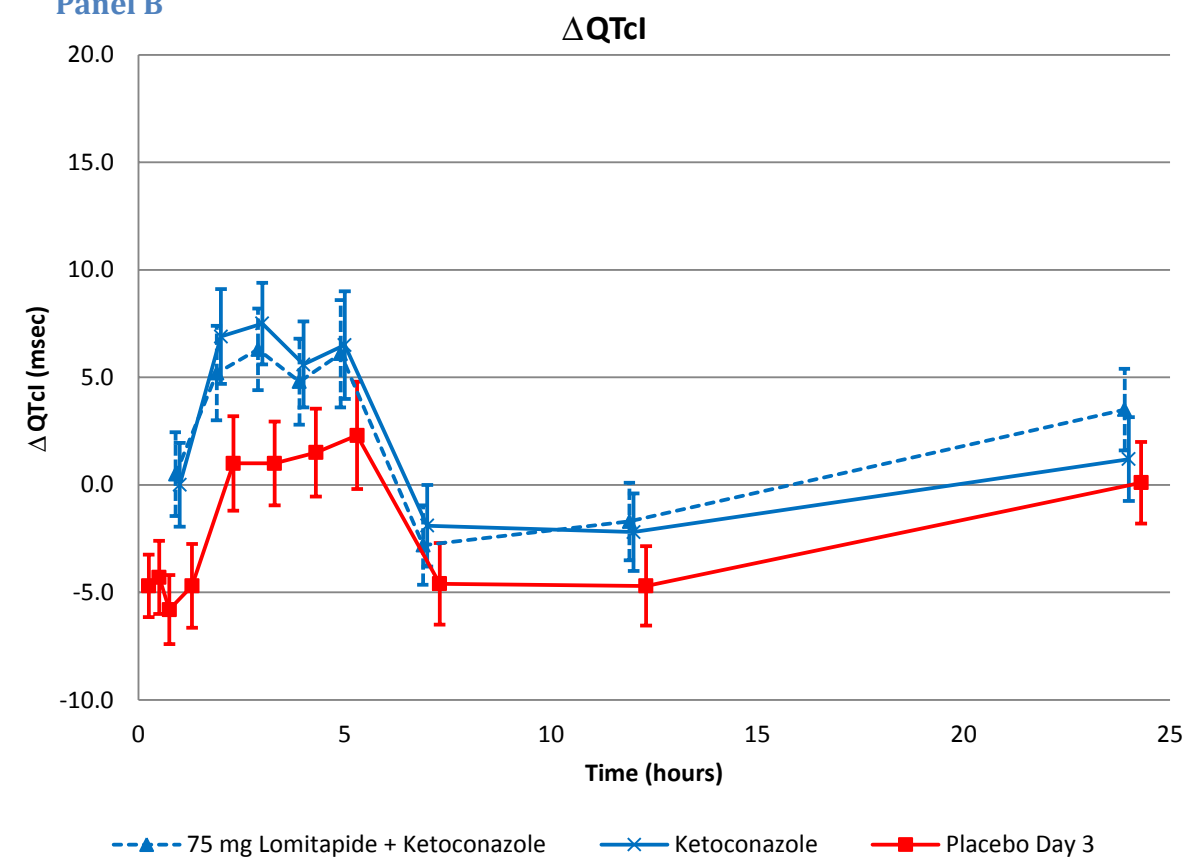

Figure 3. OTcl: Change from baseline $(\Delta \mathrm{QTCl})$ across treatment and time points. $\Delta \mathrm{QTCl}$ after dosing with lomitapide at 75 and $200 \mathrm{mg}$ was overlapping with the placebo-response. $\Delta$ QTCl was mildly prolonged when ketoconazole was given alone or in combination with lomitapide, whereas the prolongation was more pronounced after moxifloxacin. Results from the statistical modeling with the exception of the three early time points for placebo on Day 3 and moxifloxacin, which are based on summary statistics. 
Table 3. $\Delta \Delta \mathrm{OTCl}$ Across Treatments and Time points Postdosing (mean $\pm 90 \% \mathrm{Cl})^{\mathrm{a}}$

\begin{tabular}{|c|c|c|c|c|c|c|c|c|}
\hline \multirow[b]{3}{*}{ Time } & & & \multicolumn{2}{|c|}{$90 \% \mathrm{CI}$} & \multirow[b]{2}{*}{ Mean } & \multirow[b]{2}{*}{ SE } & \multicolumn{2}{|c|}{$90 \% \mathrm{CI}$} \\
\hline & Mean & SE & Lower & Upper & & & Lower & Upper \\
\hline & \multicolumn{4}{|c|}{75 mg lomitapide adjusted for placebo } & \multicolumn{4}{|c|}{$200 \mathrm{mg}$ lomitapide adjusted for placebo } \\
\hline $\begin{array}{l}1 \\
2 \\
3 \\
4 \\
5 \\
7 \\
12 \\
24\end{array}$ & $\begin{array}{r}-1.2 \\
-1.2 \\
-2.1 \\
-1.8 \\
-1.8 \\
-2.6 \\
-1.0 \\
1.4\end{array}$ & $\begin{array}{l}0.8 \\
0.8 \\
0.9 \\
1.0 \\
1.0 \\
1.2 \\
0.9 \\
0.9\end{array}$ & $\begin{array}{l}-2.5 \\
-2.5 \\
-3.6 \\
-3.5 \\
-3.4 \\
-4.6 \\
-2.5 \\
-0.2\end{array}$ & $\begin{array}{r}0.2 \\
0.1 \\
-0.5 \\
-0.1 \\
-0.2 \\
-0.5 \\
0.5 \\
3.0\end{array}$ & $\begin{array}{r}1.8 \\
0.3 \\
1.9 \\
-0.1 \\
-0.1 \\
1.3 \\
2.8 \\
1.0\end{array}$ & $\begin{array}{l}1.5 \\
1.4 \\
1.5 \\
1.3 \\
1.3 \\
1.2 \\
1.2 \\
1.3\end{array}$ & $\begin{array}{r}-0.7 \\
-2.0 \\
-0.5 \\
-2.2 \\
-2.2 \\
-0.8 \\
0.9 \\
-1.1\end{array}$ & $\begin{array}{l}4.2 \\
2.6 \\
4.3 \\
2.1 \\
2.1 \\
3.3 \\
4.7 \\
3.1\end{array}$ \\
\hline Time & \multicolumn{4}{|c|}{$\begin{array}{l}75 \text { mg lomitapide + ketoconazole } \\
\text { adjusted for ketoconazole }\end{array}$} & \multicolumn{4}{|c|}{ Ketoconazole adjusted for placebo } \\
\hline $\begin{array}{l}1 \\
2 \\
3 \\
4 \\
5 \\
7 \\
12 \\
24\end{array}$ & $\begin{array}{r}0.4 \\
-1.7 \\
-1.2 \\
-0.8 \\
-0.4 \\
-0.8 \\
0.6 \\
2.3\end{array}$ & $\begin{array}{l}1.5 \\
1.4 \\
1.5 \\
1.3 \\
1.3 \\
1.2 \\
1.2 \\
1.2\end{array}$ & $\begin{array}{r}-2.1 \\
-4.0 \\
-3.6 \\
-2.9 \\
-2.5 \\
-2.9 \\
-1.4 \\
0.3\end{array}$ & $\begin{array}{l}2.9 \\
0.6 \\
1.2 \\
1.4 \\
1.6 \\
1.2 \\
2.5 \\
4.4\end{array}$ & $\begin{array}{l}4.7 \\
5.9 \\
6.5 \\
4.1 \\
4.2 \\
2.7 \\
2.4 \\
1.1\end{array}$ & $\begin{array}{l}1.5 \\
1.4 \\
1.5 \\
1.3 \\
1.2 \\
1.2 \\
1.2 \\
1.2\end{array}$ & $\begin{array}{r}2.2 \\
3.6 \\
4.1 \\
2.0 \\
2.2 \\
0.7 \\
0.5 \\
-0.9\end{array}$ & $\begin{array}{l}7.2 \\
8.2 \\
8.9 \\
6.2 \\
6.3 \\
4.7 \\
4.3 \\
3.1\end{array}$ \\
\hline Time & \multicolumn{4}{|c|}{ Moxifloxacin adjusted for placebo } & & & & \\
\hline $\begin{array}{l}0.25^{b} \\
0.5^{b} \\
0.75^{b} \\
1 \\
2 \\
3 \\
4 \\
5 \\
7 \\
12 \\
24\end{array}$ & $\begin{array}{r}-0.3 \\
6.8 \\
10.4 \\
12.4 \\
10.7 \\
11.6 \\
10.3 \\
8.2 \\
10.0 \\
10.9 \\
6.4\end{array}$ & $\begin{array}{l}1.4 \\
2.0 \\
1.5 \\
1.5 \\
1.4 \\
1.5 \\
1.3 \\
1.3 \\
1.2 \\
1.1 \\
1.2\end{array}$ & $\begin{array}{r}-2.7 \\
3.5 \\
7.9 \\
9.9 \\
8.4 \\
9.2 \\
8.2 \\
6.2 \\
8.0 \\
9.0 \\
4.4\end{array}$ & $\begin{array}{r}2.1 \\
10.2 \\
12.9 \\
14.9 \\
13.0 \\
14.0 \\
12.4 \\
10.3 \\
12.0 \\
12.8 \\
8.5\end{array}$ & & & & \\
\hline
\end{tabular}

a Based on statistical modeling. ${ }^{\text {b}}$ Post hoc analysis based on summary statistics.

\section{ER Analysis}

The goodness-of-fit plots in Figure 4 show that the predicted $\Delta \Delta$ QTCI values were close to the observed values and it was therefore concluded that the proposed model provided an acceptable representation of the relationship between $\Delta \Delta \mathrm{QTCI}$ and plasma concentrations of lomitapide and ketoconazole. A concentration dependent effect of lomitapide was identified with a slope of 0.0258 $\mathrm{ms} / \mathrm{ng}$ per $\mathrm{mL}(\mathrm{P}=0.0771$; Table 4$)$. Based on the concentration-effect analysis, $\Delta \Delta$ QTcI can be predicted to $-0.36 \mathrm{~ms}(90 \% \mathrm{CI}:-1.47-0.76), 0.87 \mathrm{~ms}$ $(-0.77-2.52)$, and to $1.56 \mathrm{~ms}(-0.60-3.73)$ at the observed geometric mean $\mathrm{C}_{\max }$ plasma levels after dosing with lomitapide 75,200 , and $75 \mathrm{mg}$ with ketoconazole (with ketoconazole concentration set to zero in the model). The concentrationQTc relationship for ketoconazole was highly significant with a slope of $0.0013 \mathrm{~ms} / \mathrm{ng}$ per $\mathrm{mL}$ $(\mathrm{P}<0.0001)$ and an interaction between the two drugs was seen with a coefficient of -0.000006 $\mathrm{ms} / \mathrm{square}$ ng per $\mathrm{mL}(\mathrm{P}=0.038$; Table 4$)$. The adequacy of the model, as deemed from the standardized residuals versus the fitted values, the normal Q-Q plots of the standardized residuals and the random effects, was within acceptable ranges. 


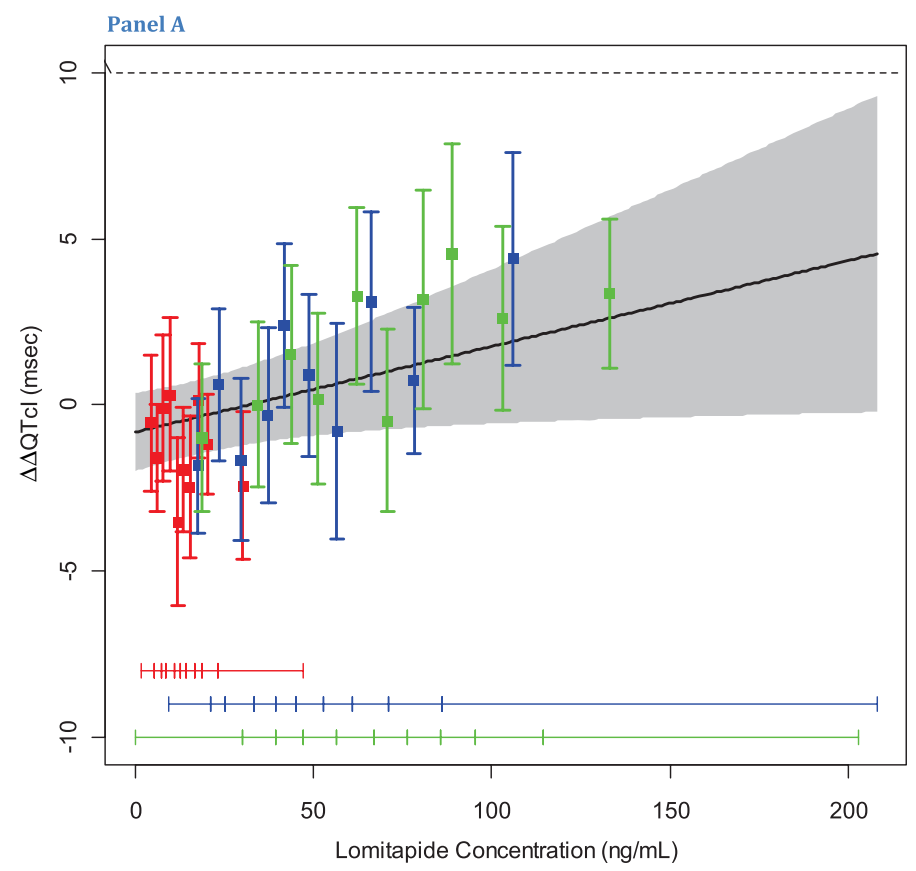

75 mg Lomitapide

$75 \mathrm{mg}$ Lomitapide + ketoconazole

- Mean $(90 \% \mathrm{Cl})$ predicted QTcl prolongation

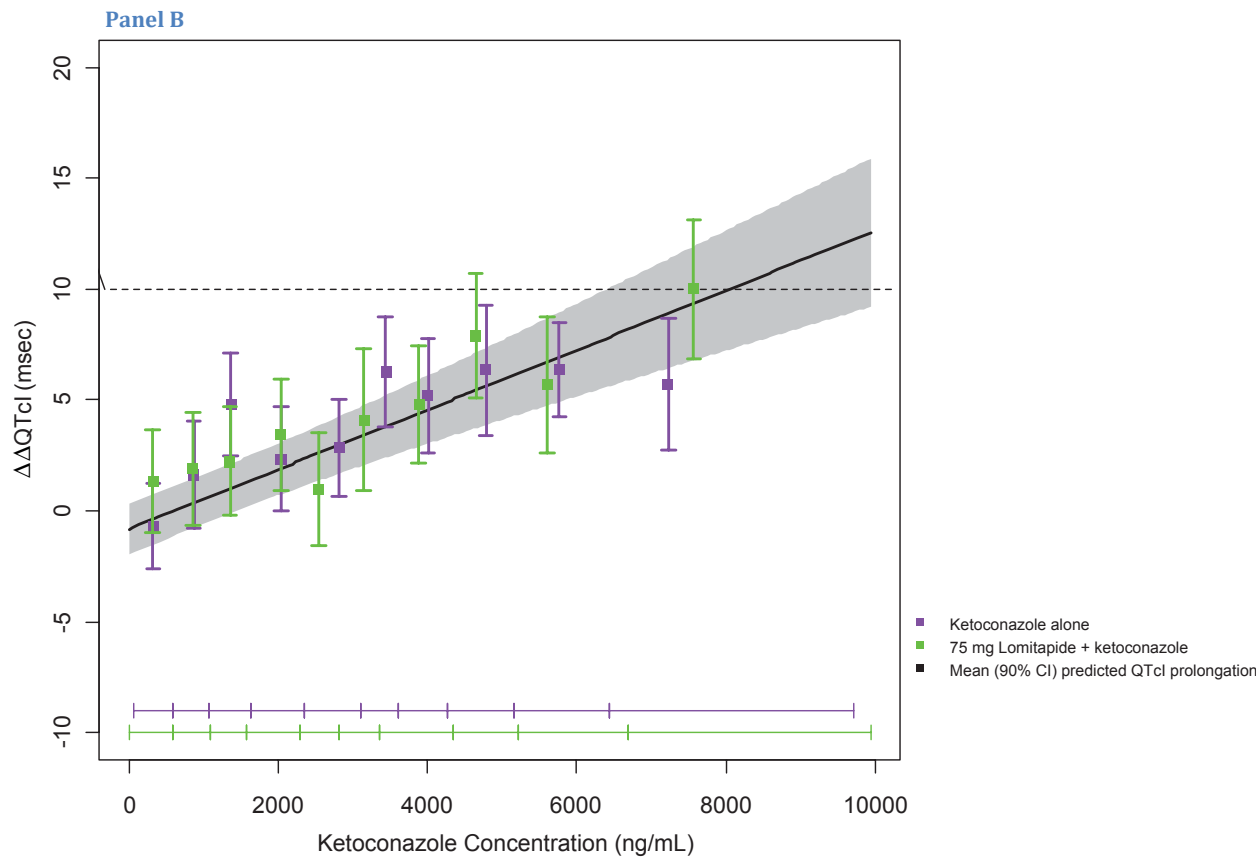

Figure 4. Goodness-of-fit plot for observed and predicted relation between lomitapide plasma levels and $\Delta \Delta \mathrm{QTCl}$ of lomitapide (panel $A$ ) and ketoconazole (panel $B$ ). The modelpredicted effect on QTcl (mean $\Delta \Delta$ QTcl with $90 \% \mathrm{Cl}$ ) is shown as a solid black line with gray shaded. Colored vertical bars denote observed mean $\Delta \Delta \mathrm{QTCl}$ with $90 \% \mathrm{Cl}$ within each plasma concentration deciles; colored horizontal lines with notches show the range of plasma concentrations for each dose within each decile. 
A.N.E. • November 2013 • Vol. 18, No. 6 • Darpo, et al., • Lomitapide TQT Study • 587

Table 4. Exposure-Response Analysis of Plasma Concentrations of Lomitapide and Ketoconazole and $\Delta \Delta \mathrm{QTCl}$

\begin{tabular}{|c|c|c|c|}
\hline Parameter & Estimate $(90 \% \mathrm{CI})$ & P Value & Between-Subject Variation \\
\hline Intercept (ms) & $-0.82(-1.99 ; 0.35)$ & 0.2504 & 4.43 \\
\hline $\begin{array}{l}\text { Slope for lomitapide } \\
\text { (m/ng per } \mathrm{mL})\end{array}$ & $0.0258(0.0018 ; 0.050)$ & 0.0771 & 0.0795 \\
\hline $\begin{array}{l}\text { Slope for ketoconazole } \\
\text { (ms/ng per } \mathrm{mL} \text { ) }\end{array}$ & $0.0013(0.0010 ; 0.0017)$ & $<0.0001$ & 0.0012 \\
\hline $\begin{array}{l}\text { Interaction of lomitapide- } \\
\text { ketoconazole } \\
\text { concentrations } \\
\text { (ms/square ng per mL) }\end{array}$ & $-0.000006(-0.000011 ;-0.000001)$ & 0.0378 & 0.000010 \\
\hline Residual variability (ms) & 7.03 & & \\
\hline
\end{tabular}

\section{DISCUSSION}

A total of 24 studies have been conducted to date with lomitapide, including Phase 1,2, and 3 studies conducted in healthy adults, adults with $\mathrm{HoFH}$, and adults with polygenic hypercholesterolemia. Phase 1 and 2 studies provided consistent evidence of statistically and clinically significant reductions in LDL-C and other lipid parameters. The most common unwanted effects are predicted based on inhibition of MTP and have been related to interference with triglyceride absorption from the gut, and accumulation of triglyceride in the liver. A Phase 3 study in 29 patients with $\mathrm{HoFH}$ formed the basis for a successful application to the FDA for approval to treat adult patients with HoFH.

This TQT study evaluated lomitapide at therapeutic and supratherapeutic plasma levels. Supratherapeutic levels were achieved two ways: (1) a high dose, $200 \mathrm{mg}$, of lomitapide in solution was given with a light breakfast, and (2) a therapeutic dose, $75 \mathrm{mg}$, was given with inhibition of the main metabolic pathway, CYP $3 \mathrm{~A} 4$ by coadministration of ketoconazole. Although a high dose will result in high plasma levels of parent and metabolites, the metabolic inhibition will result in substantially higher levels of the parent compound without a concurrent level of metabolites. A ketoconazole-lomitapide interaction study in healthy volunteers has shown that when CYP3A4 was completely inhibited, lomitapide peak plasma levels increased 15-fold after administration of a single oral dose of $60 \mathrm{mg}$ as a capsule. Although use of moderate or strong CYP $3 \mathrm{~A} 4$ inhibitors is contraindicated with lomitapide, coadministration with ketoconazole was used to determine lomitapide's effect in this potential high clinical exposure scenario.
Lomitapide given as a solution and with a meal results in higher plasma levels as compared to the capsule and the fasted state; the solution doubles the levels as compared to the capsule and a food-effect study has demonstrated that a low- or high-fat meal also resulted in significant elevations of peak plasma levels. These measures were therefore undertaken to further ensure that the peak plasma levels in the TQT study substantially exceeded those seen in patients. Lomitapide and its two inactive major metabolites have relatively long half-lives (24-28 hours for parent and approximately 21 hours for metabolites) and there is therefore an accumulation of peak plasma levels, which is consistent with the halflife and once-daily dosing. Multiple dosing with $50 \mathrm{mg}$ as a capsule results in mean lomitapide peak plasma levels of around $8.5 \mathrm{ng} / \mathrm{mL}$ and a single dose of $75 \mathrm{mg}$ in solution, given with a light meal, was expected to generate similar levels; in fact, the mean $\mathrm{C}_{\max }$ after the therapeutic dose in this study was more than twice as high $(18 \mathrm{ng} / \mathrm{mL})$ and 11fold higher $(92 \mathrm{ng} / \mathrm{mL})$ with coadministration with ketoconazole.

Lomitapide did not have an effect on the heart rate and consequently, both QTcI and QTcF appropriately corrected for heart rate changes with QTcI producing slightly lower absolute subject-specific QTc/RR slopes; QTcI was therefore selected as the primary end point, but it should be noted that results were entirely consistent with both methods. A single dose of 75 or $200 \mathrm{mg}$ lomitapide caused very small changes of $\Delta$ QTcI across all time points and when corrected for placebo, $\Delta \Delta$ QTcI did not exceed $3 \mathrm{~ms}$ at any time points postdosing. The UB of the $90 \%$ CI did not exceed $3.0 \mathrm{~ms}$ and $4.7 \mathrm{~ms}$, respectively. Lomitapide in combination with ketoconazole caused a largest mean $\Delta \Delta$ QTcI effect of 2.3 (UB: 4.4) ms (24 hours after dosing), 
when adjusted for the effect of ketoconazole alone. The QTc effect predicted by the exposure response analysis demonstrates that lomitapide at plasma levels reaching up to approximately 20fold higher levels than the mean therapeutic levels (170 $\mathrm{ng} / \mathrm{mL}$ vs $8.5 \mathrm{ng} / \mathrm{mL}$ ) will have an estimated QTc effect clearly below $5 \mathrm{~ms}$ with an UB of the CI below $10 \mathrm{~ms}$. The interaction between ketoconazole and lomitapide was significant in this model and indicated a small (slope -0.000006 $\mathrm{ms} / \mathrm{square} \mathrm{ng}$ per $\mathrm{mL}$ ) underestimation of the lomitapide QTc effect when obtaining it by directly subtracting the ketoconazole QTc effect from lomitapide in combination with ketoconazole, which can be estimated to $3 \mathrm{~ms}$ at peak plasma levels of both drugs. Even if this is taken into consideration, lomitapide at very high plasma levels does not impact cardiac repolarization in a clinically meaningful manner.

Because the lomitapide plasma levels achieved in this study mimic exposures that would only be expected to occur following contraindicated use of lomitapide (e.g., with strong CYP3A4 inhibitors), it can be concluded lomitapide will not cause QTc prolongation in patients; the study clearly represents a negative TQT study. ${ }^{1,4}$

Moxifloxacin and ketoconazole (alone or in combination with lomitapide) caused an increase of mean $\Delta$ QTcI, both with a peak effect at 3 hours postdosing (Fig. 3, Panel A and B). $\Delta \Delta$ QTcI after dosing of moxifloxacin confirmed the study's ability to detect a small QTc effect of around $5 \mathrm{~ms}$, the threshold of regulatory concern. The peak mean $\Delta \Delta$ QTCI of $12.4 \mathrm{~ms}$ was observed 1 hour after administration and the lower bound of the $90 \%$ CI (adjusted for multiplicity) exceeded $5 \mathrm{~ms}$ at all prespecified time points $(1,2,3$, and 4 hours). Since the peak $\Delta \Delta$ QTcI effect was observed at the earliest of the prespecified time points (1 hour), a rising phase was lacking and it can be debated whether this type of response confirms a drug-induced change. Additional, earlier time points $(0.25,0.5$, and 0.75 hours) were therefore analyzed. Because these were not prespecified, the subject's position at these time points was not standardized or controlled. Within this limitation, the results clearly showed the presence of a rising phase, thereby lending further support for the demonstration of assay sensitivity. The peak and time course of the moxifloxacin QTc effect were comparable to those observed in other similar studies $^{16}$ and the study therefore met ICH E14's requirements on demonstration of assay sensitivity in a TQT study. ${ }^{1,4}$

Ketoconazole-alone caused a clear QTc prolongation, which amounted to approximately $6.5 \mathrm{~ms}(\Delta \Delta \mathrm{QTcF} ; \mathrm{CI}: 4.1-8.9 \mathrm{~ms}) 3$ hours postdose after administration during 2 days of $400 \mathrm{mg}$ daily. Ketoconazole is a relatively potent hERG blocker, ${ }^{18}$ causes QT prolongation in animals ${ }^{19}$ and yet has been very rarely associated with proarrhythmias; ${ }^{20}$ (see alsohttp://www.azcert.org/medical-pros/drug-lists/ drug-lists.cfm). The QT prolonging propensity of ketoconazole has been observed in several previous studies that have incorporated a careful QT assessment. Chaikin describes two druginteraction studies using the same design with antihistamines, ebastine and loratadine, both CYP 3A4 substrates, and ketoconazole. ${ }^{6}$ Both studies were of parallel design and one treatment group $(\mathrm{n}=26$ and $\mathrm{n}=30$ ) received ketoconazole $400 \mathrm{mg}$ daily plus placebo for 8 days. On the last day of dosing, the mean change-from-baseline QTcI $(\Delta \mathrm{QTcI})$ was $6.96 \mathrm{~ms}(95 \% \mathrm{CI}: 3.31-10.62)$ and $7.52 \mathrm{~ms}$ (95\% CI: 4.15-10.89) in the ebastine and loratadine study, respectively. This observation was confirmed in a TQT study, which is detailed in a methodology article comparing different techniques for interval measurements from three separate studies. ${ }^{7}$ In one of these, $800 \mathrm{mg}$ ketoconazole as a single-dose was used as a positive control for an NCE within the same therapeutic class. The publication gives results from this study separated by site, but in an analysis of the entire group ( $\mathrm{n}=80$; data on file), $\Delta \Delta \mathrm{QTcF}$ after ketoconazole is significantly increased from 1 to 12 hours postdosing, with a peak effect of $12.5 \mathrm{~ms}$ at 3 hours. This relatively high QTc effect can most likely be explained by the higher dose of ketoconazole. Three more recent studies have confirmed the QT effect of ketoconazole; in an interaction study with cinitapride, ketoconazole $400 \mathrm{mg}$ daily for 7 days caused a mean $\Delta$ QTc effect of $6.45 \mathrm{~ms}$ (SEM 3.68). ${ }^{21}$ In a study with casopitant, ${ }^{22}$ ketoconazole $400 \mathrm{mg}$ daily for 6 days caused a largest increase of mean $\triangle \mathrm{QTcF}$ of $6-7$ $\mathrm{ms}$ and in a study with domperidone, ketoconazole $400 \mathrm{mg}$ daily for 7 days caused a largest difference versus placebo QTcF of $13.6 \mathrm{~ms}$ (95\% CI: 5.4-21.8 $\mathrm{ms})$ in men and $3.6 \mathrm{~ms}(-5.1-12.3)$ in women after 7 days. $^{23}$

Few published studies have evaluated the exposure response relationship for the QTc effect 
caused by ketoconazole; interestingly, in the study on domperidone, ${ }^{23}$ the slope of the ketoconazole concentration/QTc relationship was similar to the observed value in this TQT study: $1.6 \mathrm{~ms}$ and $1.9 \mathrm{~ms} / \mu \mathrm{g}$ per $\mathrm{mL}$ in men and women, respectively, compared to $1.3 \mathrm{~ms} / \mu \mathrm{g}$ per $\mathrm{mL}$ in this study. A similar concentration/QTc relationship was also found in the case report by $\mathrm{Zhu}$ et al., ${ }^{9}$ which discussed the implications of using metabolic inhibitors in TQT studies. Despite the inherent limitations in comparing different end points (largest vs mean effect; $\Delta \mathrm{QTc}$, placebo-corrected $\Delta \mathrm{QTcF}$ and difference vs placebo QTcF), it seems clear that ketoconazole dosed at $400 \mathrm{mg}$ daily and higher has an effect on the QTc interval, when carefully studied. In this respect, it can therefore be claimed that the sensitivity of this study to detect a small QTc prolongation was confirmed by both the ketoconazole and the moxifloxacin QTc response.

In summary, lomitapide at plasma levels clearly exceeding those that can be observed in patients dosed according to the label does not have a meaningful effect on cardiac repolarization, measured as the QTc interval. Both moxifloxacin and ketoconazole confirmed the study's sensitivity to demonstrate small drug-induced QTc effects.

\section{REFERENCES}

1. ICH Harmonized Tripartite Guideline E14. The clinical evaluation of QT/QTc interval prolongation and proarrhythmic potential for non-antiarrhythmic drugs. 2005; http://www.ich.org/cache/compo/276-254-1.html [Accessed August 2013].

2. Darpo B. The thorough QT/QTc study 4 years after the implementation of the ICH E14 guidance. Br J Pharmacol 2010;159:49-57.

3. Shah RR. Drugs, QT. Interval prolongation and ICH E14: The need to get it right. Drug Saf 2005;28:115-25.

4. ICH E14 Questions \& Answers. 2012; http:// www.ich.org/fileadmin/Public_Web_Site/ICH Products/Guidelines/Efficacy/E14/E14_Q_As_R1_step4.pdf [Accessed August 2013].

5. Stoch SA, Friedman E, Maes A, et al. Effect of different durations of ketoconazole dosing on the single-dose pharmacokinetics of midazolam: Shortening the paradigm. J Clin Pharmacol 2009;49:398-406.

6. Chaikin P, Gillen MS, Malik M, et al. Co-administration of ketoconazole with $\mathrm{H} 1$-antagonists ebastine and loratadine in healthy subjects: Pharmacokinetic and pharmacodynamic effects. Br J Clin Pharmacol 2005;59:346-354.

7. Darpo B, Agin M, Kazierad DJ, et al. Man versus machine: Is there an optimal method for QT measurements in thorough QT studies? J Clin Pharmacol 2006;46:598-612.
8. Tyl B, Kabbaj M, Azzam S, et al. Lack of significant effect of bilastine administered at therapeutic and supratherapeutic doses and concomitantly with ketoconazole on ventricular repolarization: Results of a thorough QT study (TQTS) with QT-concentration analysis. J Clin Pharmacol 2012;52:893903.

9. Zhu H, Wang Y, Gobburu JV, et al. Considerations for clinical trial design and data analyses of thorough QT studies using drug-drug interaction. J Clin Pharmacol 2010;50:1106-11.

10. Darpo B, Fossa AA, Couderc JP, et al. Improving the precision of QT measurements. Cardiol J 2011;18:401410.

11. Couderc JP, Garnett C, Li M, et al. Highly automated QT measurement techniques in 7 thorough QT studies implemented under ICH E14 guidelines. Ann Noninvasive Electrocardiol 2011;16:13-24.

12. Tornoe CW, Garnett $\mathrm{CE}$, Wang $\mathrm{Y}$, et al. Creation of a knowledge management system for QT analyses. J Clin Pharmacol 2011;51:1035-1042.

13. Tsong Y, Yan LK, Zhong J, et al. Multiple comparisons of repeatedly measured response: Issues of validation testing in thorough QT/QTc clinical trials. J Biopharm Stat 2010;20:654-664.

14. Zhang J, Machado SG. Statistical issues including design and sample size calculation in thorough QT/QTc studies. J Biopharm Stat 2008;18:451-467.

15. Hochberg Y, Benjamini Y. More powerful procedures for multiple significance testing. Stat Med 1990;9:811-818.

16. Florian JA, Tornoe CW, Brundage $R$, et al. Population pharmacokinetic and concentration-QTc models for moxifloxacin: Pooled analysis of 20 thorough QT studies. J Clin Pharmacol 2011;51:1152-1162.

17. Garnett CE, Beasley N, Bhattaram VA, et al. ConcentrationQT relationships play a key role in the evaluation of proarrhythmic risk during regulatory review. J Clin Pharmacol 2008;48:13-18.

18. Dumaine R, Roy ML, Brown AM. Blockade of HERG and Kv1.5 by ketoconazole. J Pharmacol Exp Ther 1998;286:727-735.

19. Hamlin RL, Kijtawornrat A, Keene BW, et al. QT and RR intervals in conscious and anesthetized guinea pigs with highly varying RR intervals and given QTc-lengthening test articles. Toxicol Sci 2003;76:437-442.

20. Mok NS, Lo YK, Tsui PT, et al. Ketoconazole induced torsades de pointes without concomitant use of QT intervalprolonging drug. J Cardiovasc Electrophysiol 2005;16:13751377.

21. Robert $M$, Salva $M$, Segarra $R$, et al. The prokinetic cinitapride has no clinically relevant pharmacokinetic interaction and effect on QT during coadministration with ketoconazole. Drug Metab Dispos 2007;35:11491156.

22. Johnson BM, Adams LM, Zhang K, et al. Ketoconazole and rifampin significantly affect the pharmacokinetics, but not the safety or QTc interval, of casopitant, a neurokinin1 receptor antagonist. J Clin Pharmacol 2010;50:951959.

23. Boyce MJ, Baisley KJ, Warrington SJ. Pharmacokinetic interaction between domperidone and ketoconazole leads to QT prolongation in healthy volunteers: A randomized, placebo-controlled, double-blind, crossover study. Br J Clin Pharmacol 2012;73:411-421. 